Mots. Les langages du politique

\title{
Du Gran Líder Stroessner (1954-1989) au Karai Tendota Nicanor Duarte (2003-2006)
}

\section{Capucine Boidin}

\section{Q OpenEdition}

\section{Journals}

Édition électronique

URL : https://journals.openedition.org/mots/1128

DOI : $10.4000 /$ mots. 1128

ISSN : 1960-6001

Éditeur

ENS Éditions

\section{Édition imprimée}

Date de publication : 1 novembre 2007

Pagination : 11-22

ISBN : 978-2-84788-113-4

ISSN : 0243-6450

Référence électronique

Capucine Boidin, « Du Gran Líder Stroessner (1954-1989) au Karai Tendota Nicanor Duarte (2003-2006) », Mots. Les langages du politique [En ligne], 85 | 2007, mis en ligne le 01 novembre 2009 consulté le 23 avril 2022. URL : http://journals.openedition.org/mots/1128 ; DOI : https://doi.org/ $10.4000 /$ mots. 1128

\section{(C) ENS Éditions}




\title{
Du Gran Líder Stroessner (1954-1989) au Karai Tendota Nicanor Duarte (2003-2006)*
}

\author{
- Lo que quiero decir es que el Paraguay \\ necesita un tendota. \\ - ¿Qué es « tendota»? \\ - Ha péa he’ise «motenondehára» (el que \\ muestra el camino) \\ - ¿Un dictador? \\ Asunción, 17 avril $2003^{1}$.
}

2003. Les campagne présidentielles de Nicanor Duarte Frutos (ANR, Parti Colorado) et de Yoyito Franco (Parti libéral) font rage ${ }^{2}$. Un mot achoppe dans la presse nationale: tendota. Un mot guarani au sens incertain - «chef», "guide», "président», mais aussi «sanglier dominant». Ne serait-ce pas un substitut approximatif de caudillo, rendu illégitime par l'usage répété qu'en fit le général Stroessner pendant 35 ans (1954-1989)? Comment les mots pour désigner les

* Je remercie vivement Luc Capdevila, Adelina Pusineri, Guido Rodriguez Alcalà et Bartomeu Melià pour leurs relectures critiques et leurs commentaires.

1. Nicanor Duarte Frutos, entretien avec Hugo Ruiz Olazar, abcdigital, www.abc.com.py. Voir traduction infra.

2. L'histoire de ces deux partis, l'ANR (Asociación nacional republicana, ou Parti Colorado) et le PLRA (Partido liberal radical auténtico, ou Parti libéral radical authentique) est relativement récente. Ils furent créés en 1887 afin de faire fonctionner la nouvelle Constitution démocratique de 1870, calquée sur celle de l'Argentine suite à la guerre que mena et perdit le Paraguay contre la Triple alliance formée par le Brésil, l’Uruguay et l'Argentine (1864-1870). Si le Parti Colorado domine de 1887 à 1904, à partir de cette date et jusqu'en 1932, le Parti libéral est hégémonique : l'alternance au pouvoir est le résultat de «révolutions» armées menées par différentes fractions du Parti libéral. La guerre du Chaco, qui oppose le Paraguay à la Bolivie (1932-1935), entraine l'irruption des militaires dans le champ politique. Elle culmine avec le coup d'État du général Stroessner en 1954. Il remplace les colonels par des officiers moins gradés qui devaient leur promotion à Stroessner. Il les encourage à la corruption et au commerce illégal ainsi qu'à l'administration de commerces légaux pour blanchir leur argent tout en exigeant leur affiliation au Parti Colorado. Ces officiers, souvent issus de la classe paysanne, achetaient une estancia, base du pouvoir symbolique (Fregosi, 1997, p. 100-101). Depuis lors, malgré la démocratie instaurée suite au coup de force du général Rodriguez - gendre de Stroessner -, le Parti Colorado reste au pouvoir pour la soixantième année consécutive.

Université Paris 3 (IHEAL, CREDAL), INALCO, capucine.boidin@gmail.com 
responsables politiques ont-ils évolué en espagnol et en guarani depuis la mise en place de la démocratie?

L'usage du guarani, sous la forme de mots clés et de «petites » phrases, mais aussi de longs discours, est récurrent en politique paraguayenne. Les rassemblements ruraux et suburbains, les slogans télévisés, et plus récemment les écrits - tracts, affiches, articles de presse - mêlent le guarani et l'espagnol. Impossible de mener une campagne efficace sans manier l'oralité guarani : le Paraguay est un pays guaranophone à $90 \%$ (50\% de bilingues guarani espagnol et $40 \%$ de monolingues guarani). La Constitution de 1992 donne un statut similaire à l'espagnol et au guarani. Le système éducatif commence timidement à devenir bilingue. Pourtant, seuls $2 \%$ de la population se définissent indiens. Cas unique en Amérique latine, le guarani, langue générale avant la conquête espagnole, est devenu celle des Métis et des Créoles à l'époque coloniale avant de devenir un symbole d'identité nationale. Les mots du pouvoir puisent autant dans les lexiques espagnol que guarani. Pour suivre leurs usages récents, nous travaillerons sur deux corpus de nature et d'échelle très différentes : la presse nationale et internationale consultable sur Internet nous donnera accès à la manière dont se disent les relations de pouvoir à distance et médiatisées. L'observation ethnographique des interactions politiques quotidiennes et la réalisation de trois entretiens ${ }^{3}$ en guarani avec des chefs des sous-sections locales des partis nationaux permettront de suivre l'évolution des mots de l'autorité dans les relations interpersonnelles. Comment la population désigne-t-elle ses leaders locaux (hommes et femmes)? Comment s'autodésignent-ils? Et leurs supérieurs, comment nomment-ils leurs subalternes? Dans quelle langue? Dans quelle situation?

\section{Karai tendota dans la presse nationale et internationale}

La défense et l'illustration du guarani sont soutenues par différents acteurs et courants : depuis les années trente par le populisme, autoritaire et militaire ${ }^{4}$, et depuis les années quatre-vingt-dix par le multiculturalisme, qui cherche à promouvoir une image bilingue et biculturelle de la communauté nationale (Boidin, 1999). L'usage du guarani par Nicanor s'inscrit-il plutôt dans les us et coutumes

3. Terrains répartis entre 1999 et 2005 dans deux hameaux, ici codés Isla et Tatu. Le premier entretien, codé Labri, est une suite de conversations menées en 1999 avec un ex-caudillo du Parti libéral âgé de 75 ans. Refusant d'être enregistré, il dictait lentement ses propos afin de me permettre de les noter. Le deuxième, codé Claudio, réalisé en 2000 à l'initiative de l'homme luimême, la quarantaine, président de la sous-section Colorado, est un monologue expliquant sa prise de pouvoir aux dépens de l'ancienne présidente. Le dernier, codé Kelo, a été enregistré en 2005 avec cette femme, d'environ 40 ans, sur mon initiative. L'intégralité des entretiens avec traduction française est consultable dans les annexes de ma thèse (Boidin, 2004).

4. Le général Stroessner déclara le guarani langue nationale en 1967. 
conservatrices nationalistes ou dans un programme d'idées progressistes? Nicanor Duarte Frutos ne provient pas de la hiérarchie militaire : avocat, philosophe, journaliste et professeur de sociologie, il est ministre de l'Éducation de 1993 à 2003, période pendant laquelle il donne une première impulsion à l'éducation bilingue guarani-espagnol. Pourtant, l'usage qu'il en fait dans l'entretien suivant l’inscrit dans une lignée populiste :

Le journaliste: On peut penser, d'après vos paroles, que cela ne vaut pas la peine de nettoyer cette structure corrompue qui se maintient au pouvoir depuis des décennies.

Nicanor Duarte Frutos: Ce que je veux dire, c'est que le Paraguay a besoin d'un tendota 5 .

J. : Qu'est ce qu'un tendota?

N. D. F. : \{Cela veut dire : «celui qui montre le chemin ».\}

J. : Un dictateur?

N. D. F. : Non. Tendota est celui qui prend la direction des travaux avec le peuple, celui qui connait la douleur et l'espérance des gens parce qu'il les a vécues et parce qu'il vit quotidiennement avec le peuple. Ici, nous n'avons pas besoin d'un président amidonné, prisonnier de ses pactes, sans capacité de direction et sans pouvoir ${ }^{6}$.

Discours auto-instituant de la personne même du locuteur, l'analogie est ici christique 7 . Quelques lignes plus loin, l'énonciateur s'autoproclame, en guarani :

$\{$ Moi je suis le chef, je serai le chef, je suis l'exécutif\} [...] je suis le Parti Colorado

L'usage du guarani est tout à fait contrôlé et limité aux moments où, cherchant à s'adresser directement au peuple, il s'autodéfinit comme le guide idéal du Paraguay. La réponse de son adversaire ne s'est pas fait attendre, sans quitter finalement le registre christique :

J. : Pourquoi dit-il qu'il veut être le tendota?

Y. F. : Il faut souligner que le tendota, «cacique», «caudillo», «chef», n'est pas ce dont a besoin le Paraguay. Davantage qu'un tendota, le Paraguay a besoin d'un serviteur au comportement démocratique9.

Dix jours après cette intervention, Nicanor Duarte Frutos a gagné l'élection présidentielle et l'expression tendota s'est installée dans le paysage médiatique.

Curieusement, les médias internationaux ont repris l'appellation sans recul critique. Tendota est utilisé comme un équivalent neutre de président

5. Dans la suite de l'article, les textes de presse et d'entretiens traduits à partir du guarani seront mis entre accolades.

6. Nicanor Duarte Frutos, abcdigital, Asunción, 17 avril 2003.

7. Dans un dictionnaire du $17^{\mathrm{e}}$ siècle, le mot est recueilli et réinterprété par les jésuites en ce sens : «Tendotara: celui qui va devant, frère ainé [...] Devant nous va Jésus-Christ notre Seigneur, il donne l'exemple afin que nous l'imitions. » (Montoya, 1639, p. 380.)

8. Nicanor Duarte Frutos, abcdigital, Asunción, 17 avril 2003.

9. Yoyito Franco, abcdigital, Asunción, avril 2003, www.abc.com.py 
par l'Organisation pour le multilinguisme, dont le siège est à Barcelone : elle a lancé le 28 février 2006 une campagne Internet pour promouvoir l'officialisation du guarani par le Mercosur ${ }^{10}$. De fait, le 23 novembre 2006, une résolution des ministres de la Culture l'a proclamé langue officielle du Mercosur. Depuis, lors du $32^{\mathrm{e}}$ sommet des présidents du Mercosur les 18 et 19 janvier 2007, elle a été déclarée langue du Mercosur mais sans être reconnue comme langue de travail ${ }^{11}$. À Barcelone, la conférence que Nicanor a donnée le 29 octobre 2005 - «Ma langue maternelle est le guarani » - fait mention dans son titre de «Tendota Karai Nicanor $»^{12}$. Antérieurement, le $\mathrm{CIDH}$, Centre international des droits de l'homme, avait également publié une lettre en guarani sur la liberté d'expression en traduisant "président» par tendota ${ }^{13}$. Effectivement, les ouvrages de référence les plus consultés - la grammaire et le vocabulaire d'un père jésuite espagnol - donnent pour tendota: «cabecilla», "tête» d'un mouvement (Guasch, 1961, p. 61, 149, 159). L'erreur avait été relevée par un anthropologue paraguayen : tendota est, pour les paysans d'aujourd'hui, un sanglier dominant ${ }^{14}$. Tout Paraguayen a l'intuition que derrière le terme tendota se profile l'image de l'homme fort, du mbarete en guarani. Les efforts du président pour rebaptiser le palais présidentiel, mburuvicha roga, en tendota roga furent en fin de compte sans effet ${ }^{15}$. Les mouvements d'opposition le traduisent directement par «dictateur $»^{16}$. Ses agissements ne sont pas sans donner de l'eau à leur moulin : il viola la Constitution quelques heures le 14 mars 2006 en cumulant la présidence de la République et du parti ${ }^{17}$. Il y a quelques mois encore, il cherchait à faire modifier la Constitution afin de pouvoir se faire réélire une deuxième fois.

\section{Karai tendota contre mburuvicha?}

Nicanor Duarte Frutos aurait donc pu choisir de se faire appeler mburuvicha, chef. Il a préféré essayer, sans succès, de rebaptiser le palais présidentiel - mburuvicha roga - en tendota roga. Mais pourquoi cette opposition?

\footnotetext{
10. Al Presidente del Paraguay / Paraguái Rendotápe / Ao Presidente do Paraguai / To the President of Paraguay, http://www.om-plural.org/ [om : organització pel multilingüisme].

11. Résolution $\mathrm{n}^{\circ} 31$ de la déclaration des présidents, 19 janvier 2007 : http://www.presidenciamercosur.org/es/

12. www.datamex.com.py/guarani/opambae-rei

13. http://www.cidh.org/countryrep/Paraguayo1gua/cap.6.htm

14. Malheureusement, le père Antonio Guasch décéda avant de pouvoir intégrer les corrections de Leon Cadogan. Elles sont publiées dans Amerindia, $1987, \mathrm{n}^{\circ}$ 12, www.celia.cnrs.fr

15. Une voix radiophonique célèbre, Jorge Rubiani, fit remarquer que le palais présidentiel deviendrait alors une grotte de sangliers... (revistarenovación.com.ar/latinoamerica/).

16. Une marche contre "les dictateurs et tendota» a été organisée le 2 février 2006 pour commémorer le $17^{\mathrm{e}}$ anniversaire de la chute du régime dictatorial de Stroessner. Ultima Hora, 31 janvier 2006.

17. www.clacso.org.ar/
} 
Chez les Guarani, les mburuvicha étaient des guerriers renommés dont le pouvoir coercitif ne durait que le temps des guerres. En période de paix, leur autorité reposait sur un consensus et pouvait sans cesse être révoquée (Clastres, 1974). Au $17^{\mathrm{e}}$ siècle, le terme fut repris par les missionnaires pour signifier "cacique principal», charge désormais vitalice et "supérieur religieux». À partir de la racine tuvicha, et ajoutant un suffixe superlatif -ete, les jésuites créèrent le mot «roi, empereur» (Montoya, 1639, p. 400). De fait, ce terme est attesté dans les lettres écrites en 1630 par des Indiens des réductions jésuites afin de protester auprès du roi contre les mauvais traitements dont ils étaient victimes de la part des Espagnols :

Écouter les paroles de Notre Grand Supérieur - Ñande Rubichabete - [néologisme signifiant Le Roi] nous réjouit grandement et nous console... Autrefois, quand nous étions pauvres et maltraités par les Espagnols - karai -, il nous semblait que Ñande Rubichabete n'en savait rien. (Présenté et traduit par Melià, 2006.)

Que fait donc Nicanor Duarte Frutos en se faisant appeler karai tendota et non pas mburuvicha tendota, par exemple?

À l'époque préhispanique, les karai étaient les prophètes qui incitaient les Guarani à partir dans de grandes migrations à la recherche de «la terre sans mal », c'est-à-dire sans mort, ni souffrance, ni prohibitions matrimoniales (Clastres, 1975). Pierre Clastres interpréta la lutte des karai prophètes contre les mburuvicha chefs comme celle de la société contre l'État (1974). Ce qui est certain, c'est que le terme est employé très tôt à l'époque coloniale pour désigner les missionnaires et les conquistadors. Ceci contre la volonté des premiers. Selon un jésuite grammairien du $17^{\mathrm{e}}$ siècle, karai voudrait dire "astucieux, habile. Vocable qu'ils utilisaient pour honorer leurs jeteurs de sort de manière universelle. Et ainsi, ils l'appliquèrent aux Espagnols, et très improprement aux chrétiens et aux choses bénies, et c'est pourquoi nous ne l'utilisons pas en ce sens» (Montoya, 1639, p. 90).

Aujourd'hui, n'en déplaise à Montoya, karai est un lexème à tendance nominale et verbale qui signifie «baptême », «bénédiction » et «sanctification ». Che karai : «je suis baptisé». Amongarai : «je baptise». Équivalent du don/señor espagnol, il est réservé aux personnes d'un certain âge et d'une certaine réputation. Au niveau local, karai pourrait être traduit par «notable » au sens où ces derniers se trouvent au centre des réseaux d'échange et de prise de décision dans un groupe.

Autrement dit, karai a une dimension religieuse qui manque à mburuvicha. Comme le souligne Luc Capdevila, ce n'est pas un hasard si les premiers grands caudillos du $19^{\mathrm{e}}$ siècle choisirent de se faire appeler karai plutôt que mburuvicha (2007, p. 93-94). Ce terme est certes appliqué à un président à la fin du $19^{\mathrm{e}}$ siècle, mais dans un contexte militaire: dans le journal Cacique Lambaré, édité lors de la guerre de la Triple Alliance (1864-1870), le président et 
maréchal Francisco Solano López est Karai López, Karai Guasu, grand Karai, Ñande Ruvicha Guasúpe, «notre grand chef » ou Ore Ruvicha Mariscal, «notre chef maréchal $»^{18}$.

Ainsi, lorsque Nicanor Duarte Frutos se fait appeler karai tendota, il se présente moins comme un homme fort en armes que comme l'homme providentiel prophétique. En filigrane se dessine également une filiation avec le "général» Stroessner.

\section{Caudillo : entre injure et concept...}

À l'instar de Perón (Périès, 2007), Stroessner récusa le terme caudillo et préféra se faire appeler conductor, gran líder, general, primer mandatario. Dans les campagnes, il était appelé mi general, el rubio (le blond) et tembelo (allusion à sa lèvre inférieure, qui était proéminente). Il faut comprendre pourquoi le mot caudillo a mauvaise presse dans le Rio de la Plata dès le début du $19^{\mathrm{e}}$ siècle. Selon un phénomène classique, le stigmate a pu être retourné et même devenir une notion socio-historique.

Dans l'Espagne du $19^{\mathrm{e}}$ siècle, cacique et caciquismo - ce dernier terme fut repris des Tainos par Christophe Colomb et appliqué par la suite à tout chef «natif» - sont des injures qui permettent de disqualifier l'adversaire en le figeant dans un passé barbare opposé au progrès de l'État et de la démocratie représentative. Dans la péninsule ibérique, le général Franco se fait appeler el Gran Caudillo (Ruiz, 2006). Par un jeu de miroir inversé, en Amérique Latine, ce sont les caudillos et caudillaje qui fusent pour fustiger les opposants: «La presse libérale utilisa le vocable caudillo à profusion pour se référer à un type de commandement illégitime, barbare, presque toujours associé à la tyrannie ou au despotisme et qui était finalement entendu comme la dégénération américaine de l'héritage violent et intolérant du colonialisme espagnol.» (Ruiz, 2006, p. 33.) En réalité, lesdits caudillos ne devaient rien à l'héritage espagnol. Ils représentaient un phénomène structurel, propre aux situations de transition et de mise en place des États modernes. L'autorité du caudillo reposait sur trois caractéristiques : une base économique (la propriété des terres et le contrôle des ressources locales), une implantation sociale et un projet politique. Il avait derrière lui une histoire remplie d'exploits, il cherchait à obtenir puissance et richesse par les armes. Il était chargé de combler les vides du pouvoir lors des désordres de l'État, faisant du personnalisme et de la violence sa loi. Si les militaires pouvaient se transformer en caudillo et vice versa, il ne faut pas les confondre. Le caudillo était surtout celui qui se constituait une clientèle.

18. Répertoire et fac-similé mis en ligne par Wolf Lustig: http://www.staff.uni-mainz.de/lustig/ guarani/ 
Les relations patron/client étaient informelles et personnelles, fondées sur l'échange inégal de service et de loyauté. Elles suivaient le schéma propriétaire terrien/paysan. Il existe sur ce point une différence entre le $19^{\mathrm{e}}$ siècle argentin et le $19^{\mathrm{e}}$ siècle paraguayen.

Le Paraguay eut à sa tête un caudillo - le docteur Gaspard Rodríguez de Francia - qui supprima l'essentiel de l'élite et fit de l'État le propriétaire de la majeure partie des terres du pays. Il parvint ainsi à établir des liens presque directs entre sa personne et la population. Il n'y eut pour ainsi dire pas de caudillos intermédiaires. La conjoncture changea radicalement à la fin $\mathrm{du} 19^{\mathrm{e}}$ siècle et au début du $20^{\mathrm{e}}$ avec l'instauration de la démocratie libérale dans une situation d'après-guerre très troublée : elle vit se multiplier les caudillos estancieros surl'ensemble du territoire.

Sous Stroessner, le terme fut également repris pour nommer/disqualifier les autorités locales classiquement incarnées par trois figures: l'estanciero local (grand propriétaire terrien éleveur de bétail), l'acopiador (intermédiaire commercial) et le caudillo prebendario (créé par le parti). Ces caudillos traditionnels tenaient leur pouvoir de leurs différents capitaux : économique, social et symbolique. En revanche, le caudillo prebendario, par sa position dans le parti, disposait du monopole de la violence «légitime» et des ressources de l'État - postes de fonctionnaires, fonds, etc. (Yore, 1994). Ceux-ci étaient alors souvent des propriétaires moyens, de petits commerçants sans terres ou des contremaitres, installés au cœur même des hameaux. "Le Parti Colorado était une vaste machine de contrôle, mais aussi de redistribution et de promotion sociale, plus efficace à bien des égards que les partis communistes d'Europe de l'Est. » (Fregosi, 1997, p. 105-106.)

$\mathrm{Si}$ «l'autre» peut être un caudillo, le terme n'est pas revendiqué pour soi. Stroessner choisit également l'appelation de «second reconstructeur». En effet, dans l'interprétation colorado de l'histoire nationale, le docteur Gaspard Rodríguez de Francia est le «fondateur » de la nation, Don Carlos Antonio López le «constructeur» de l’État moderne, le maréchal Francisco Solano López le «défenseur» de la souveraineté nationale et le général Bernardino Caballero le "premier reconstructeur» du pays après la guerre de la Triple Alliance (Capdevila, 2007). Ni maréchal (il n'y en eut qu'un seul dans l'histoire nationale), ni général, ni mburuvicha ou conductor, Nicanor pouvait difficilement se placer autrement qu'en leader civil, en karai tendota. Un tendota qui rappelle le gran líder. Mais cette création linguistique au niveau de la présidence a-t-elle une incidence dans les campagnes de l'intérieur du pays? Comment les mots médiatisés pour dire les plus hautes autorités de l'État s'articulent-ils avec les mots du pouvoir dans les relations interpersonnelles? Existe-t-il un décalage temporel? 


\section{Disparition d'un mot et d'une fonction : les oficiales de compañía}

Depuis la fin de l'époque coloniale jusqu'au régime du général Stroessner, les hameaux, appelés compañías rurales (compagnies rurales), ont été sous l'autorité d'oficiales et de sargentos de compañía (officiers et sergents de compagnies). Ils cumulaient des fonctions militaires, policières et judiciaires. Appellations qui signalent une militarisation profonde et durable de la société : pays frontière marqué par des conflits sur tous les fronts, le Paraguay a connu la mobilisation chronique d'une proportion considérable de la population masculine du pays.

Sous le régime de Stroessner, l'oficial de compañía était avant tout un relais de la police, qu'il informait de ce qui se passait dans la compagnie. Chargé de demander des autorisations de fêtes, de rassemblements, de courses de chevaux, etc., à la police de la ville et de surveiller le bon déroulement de ces festivités, il devait empêcher ou régler les conflits. Il était choisi par les autorités policières de la ville de San Ignacio, elles-mêmes sous contrôle du Parti Colorado de Stroessner.

Avant, l'officier de compagnie était tout. Le commissaire du district venait faire une réunion et annonçait aux habitants, vecinos, qui allait être l'officier et qui allait être le sergent. Ils n'avaient pas de salaire, todo ad honorem. [...] La municipalité lui laissait le contrôle du marquage des vaches. [...] II était chargé d'aider les habitants à résoudre leurs problèmes entre eux, il les faisait parler et s'ils ne s'entendaient pas, ils allaient devant l'autorité du district. (Mecho, Isla, 2001.)

Le remplacement de ce système par une comisión vecinal après la chute de Stroessner, en 1989, est loin d'être anodin et illusoire (Boidin, 2004b). Le président de la comisión vecinal est localement élu. Cette commission récolte les impôts, a un budget et doit rendre des comptes tous les six mois. Elle contrôle des sous-groupes spécialisés dans différents domaines (Église, École, Chemin, Eau courante, Électricité...). Seule continuité avec l'officier de compagnie : la commission nomme un responsable du marquage des animaux. Le déroulement des réunions prolonge les rites d'interaction quotidiens des habitants qui s'ordonnent selon le principe du consensus tacite. Évitant les discussions frontales, ils feront toujours bonne figure les uns devant les autres, avec un art consommé qui laisse pantois l'ethnologue peu à peu instruit des multiples rumeurs et dissensions villageoises, jusqu'à ce que la tension soit «résolue» par le départ ou l'homicide. La figure policière et dictatoriale de l'officier de compagnie est donc tombée en désuétude devant la mise en place d'une institution démocratique. À partir de là, les différentes sous-commissions sont gérées par des meneurs qui ont chacun leurs réseaux extérieurs qui les soutiennent et qu'eux-mêmes appuient. Une des particularités des hameaux est de 
disposer de plusieurs "petits » chefs potentiels et de ne pas être sous la coupe d'un propriétaire terrien omnipotent. Ce système de concurrence avive certes le clientélisme mais limite l'ampleur des liens de dépendance, conforte leur marge de manœuvre pour améliorer leurs conditions de vie dans un contexte économique défavorable.

\section{Ton chef te dit caudillo}

Quoique son utilisation décline, le terme de caudillo continue à être mobilisé dans les campagnes. Probablement parce qu'il admet un sens neuf, démilitarisé. Comme en Argentine, il désigne un dirigeant local capable de réunir des votes pour un parti dans une région donnée en actionnant les leviers du clientélisme (Lynch, 1993, p. 531-532). La dimension autoritaire du terme caudillo s'estompe au profit de ses aspects clientélistes. Signe de cette érosion et de cette resémantisation, les contextes d'énonciation du terme sont désormais limités. Le mot n'est plus utilisé par les jeunes meneurs locaux (génération des quarante ans), ni pour se désigner, ni pour se référer aux dirigeants du parti. En revanche, ces derniers appellent caudillos les maillons subalternes :

Caudillo veut dire que tu attires tout, que tu tiens tout comme cela, tu bouges et derrière toi, tu as tout un groupe que tu maintiens, ton chef te dit caudillo. (Kelo, Isla, 2005.)

Caudillo devient de moins en moins un terme d'adresse - remplacé par des mots plus neutres comme jefe, dirigente, mburuvicha et karai. Mais caudillo garde une fonction descriptive et générique. Ainsi, selon Francisco, infirmier et militant actif du Parti Colorado dans une ville de l'intérieur du pays, un caudillo est une personne qui entraine derrière lui une centaine de votes, qui est responsable et auprès de qui on vient demander de l'aide. «En ce sens, dit-il, Ña Kelo, l'épouse de Martin, du hameau où tu es allée, est une caudilla, celle qui faisait le plus bouger les choses là-bas. » C'est la seule occurrence du terme caudilla au féminin que nous ayons personnellement pu relever lors de nos différents terrains conduits entre 1998 et 2005. Il est significatif qu'elle s'énonce en ville lorsque les fonctions du caudillo sont moins définies par la force, la virilité et le port d'armes que par la capacité à mobiliser l'aide sociale de l'État ou du parti au profit des coreligionarios. Lesdits caudillos, comment nomment-ils à leur tour leurs supérieurs dans le parti?

\section{Che kandidato}

Claudio, président de la section du Parti Colorado de son hameau, dans l'entretien réalisé ensemble, ne désigne jamais ses «supérieurs» au sein du parti caudillo, jefe ou dirigente, rarement líder et presidente. Il fait sans cesse 
référence à «mon candidat»: che kandidato (7 occurrences) et au «chef de mon candidat»: che kandidato ruvicha (1 occurrence). Il mobilise également la périphrase: «celui que j'appuie en politique» (la che apoja chupe politikape, 7 fois). Comme s'il était fier d'avoir parié sur le bon cheval, il m'explique les avantages qu'il retire de "son candidat» aux dernières élections. Même si ce dernier n'a pas gagné au niveau local, il a une position importante dans une entreprise nationale et ses supérieurs sont à la tête de l'État.

\section{\{- Ce sont les tiens qui sont à la gobernación?}

- Ici à San Ignacio, mon candidat, celui que j’appuie, est employé au barrage hydroélectrique de Yacireta [sous-entendu : cela permet d'obtenir des ressources économiques]. Mais à Asunción, ce sont ses leaders [líders] qui commandent, c'est le chef [mburuvicha] de mon candidat qui commande à présent. Donc maintenant on obtient beaucoup de choses avec eux, parce qu'ils commandent.

Hum, ils commandent maintenant.

Avant les nôtres ne commandaient pas, d'autres le faisaient, donc il y avait peu d'aide [sa'i la ayudo], parce que tu dois avoir de l'argent pour aider, tu ne peux pas utiliser l'argent de l'État. Maintenant, peut-être qu'il utilise l'argent de l'État, celui que j'ai soutenu. Maintenant, je peux obtenir davantage de choses avec lui, le type est venu ici, il est formidable, il est bon, et moi, comme il aide les gens je le soutiens, et s'il n'aide pas, comment je le soutiendrais? C'est pour ça qu'on s'entend maintenant. Il est membre du conseil de Yacireta, il gagne 25 millions de guarani, 8000 dollars, beaucoup d'argent.\} (Claudio, Isla, 2000.)

L'évidence «naturelle» avec laquelle est évoquée l'utilisation de l'argent de l'État et de ses entreprises par les différentes fractions du Parti Colorado n'est pas sans poser question. La circulation concrète est la suivante : Claudio décide d'inviter un candidat au poste de gouverneur à une fête organisée pour les quinze ans de la fille d'un de ses oncles. «Ill nous plaisait, c'était mon candidat à l'époque, on l'avait invité, [...] je l'ai invité et il est venu, c'est déjà mon ami le gars. » Le candidat à l'élection lui propose alors de mettre sur pied une commission pro-électrification. Claudio hésite et attend que le candidat s'engage fermement à l'appuyer avant de se lancer. Une fois montée, la commission organise des courses de chevaux pour réunir deux millions de guaranis, donnés de la main à la main au gouverneur. En échange, ce dernier débloque les ressources de l'État et la population se retrouve en dette vis-à-vis de l'administration. Mais celle-ci ne viendra pas - les assure-t-on - leur réclamer son dû (sic). Concrètement, Claudio obtient de réceptionner personnellement 6800 mètres de câble de basse tension. Fort de ce dépôt, il décide de les donner aux plus pauvres. Claudio lui-même insiste sur sa pauvreté, sur le fait qu'il donne aux plus pauvres qui sont nombreux (et sont donc un électorat important), et sur la colère des «riches » qui n'ont pas reçu de câble gratuitement.

En définitive, le bien collectif individualisable qu'est l'électricité est mis en place alors que ni le marché, ni la seule fonction redistributive de l'État ne 
l'auraient rendu possible. Personne n'est dupe dans ce jeu, ni le «candidat», ni la communauté, ni le líder, qui cherche à soutenir le candidat le plus rentable, celui qui lui permettra de ne pas passer lui-même pour un «menteur» et un « radin » auprès des siens. Signe des temps, les supérieurs au sein du parti sont des «candidats» que l'on décide d'appuyer, non pour leurs idées, certes, mais en suivant des critères rationnels. Aujourd'hui, ce qui fait un chef local, c'est moins son pouvoir coercitif et sa position officielle, donnée par ses supérieurs à l'intérieur du Parti Colorado, que sa capacité à être au centre des réseaux de clientèle et à choisir un bon candidat à soutenir. Plusieurs fractions du Parti Colorado se battent entre elles pour gagner des électeurs. Au hameau, trois hommes et une femme appuient chacun un candidat d'une fraction différente et se font concurrence. Si les candidats qu'ils appuient continuent à les appeler caudillos, la population quant à elle parle des karai et kuña karai (femme karai), un terme beaucoup plus anodin.

Se vérifie alors l'hypothèse selon laquelle le clientélisme augmente dans les phases de transition démocratique (Goirand, 1998). Le clientélisme n'est pas un archaïsme mais un attribut de notre modernité politique. Certes, le lien démocratique est censé remplacer les liens clientélistes. En réalité, la démocratie représentative se fonde en les critiquant, mais paradoxalement, les entretient (Briquet, Sawicki, 1998).

Les mots du pouvoir dans les campagnes se sont davantage démocratisés que dans les plus hautes sphères. Même si le mot caudillo présente encore quelques occurrences (de la part de la hiérarchie du parti envers ses subalternes...), les officiers et sergents de compagnie ont disparu et laissé la place aux karai líders, hommes ou femmes. En choisissant de se faire appeler Karai Tendota, Nicanor Duarte Frutos innove moins qu'il ne s'inscrit dans la tradition des hommes providentiels du Parti Colorado et de l’État.

\section{Glossaire}

Mburuvicha / ruvicha : chef, leader, président.

Karai et kuña karai : monsieur et madame, adultes nécessairement baptisés ayant formé une famille et jouissant d'une certaine respectabilité et autorité dans leurs communautés. Notables.

Tendota : guide, homme fort.

Mbarete : fort.

Guasu: grand.

-' $i$ : diminutif.

Nande, ore : première personne du pluriel, «nous » inclusif et exclusif. 


\section{Références}

BoIDIN Capucine, 1999, «La política de educación bilingüe guarani español en el Paraguay de los años 1990 », Revista paraguaya de sociología, año 36, n¹05, p. 147-158.

- 2004a, Guerre et métissage au Paraguay. Deux compagnies rurales de Misiones $(2001-1767)^{19}$, thèse de doctorat, Paris 10 Nanterre, $687 \mathrm{p}$.

- 2004b, "La démocratisation du pouvoir local au Paraguay: illusion ou réalité?», Cahier des Amériques latines, ${ }^{\circ}$ 46, 2004/2, p. 73-84.

- 2006, "Clientélisme et don contre-don au hameau (Paraguay 2000)», Politique et société en Amérique latine. Perspectives sociologiques, D. Martuccelli et al. éd., Lille, Presses du Septentrion.

BRIQUET Jean-Louis, SAWICKI Frédéric éd., 1998, Le clientélisme politique dans les sociétés contemporaines, Paris, PUF.

CAPDEVILA Luc, 2007, Une guerre totale. Paraguay, 1864-1870, Rennes, Presses universitaires de Rennes.

CLASTRES Pierre, 1974, La société contre l'État, Paris, Minuit.

CLASTRES Hélène, 1975, La terre sans mal. Le prophétisme tupi-guarani, Paris, Le Seuil.

Fregosi Renée, 1997, Le Paraguay au xxe siècle. Naissance d'une démocratie, Paris, L'Harmattan.

Galeano Luis, Yore Myriam, 1994, Poder local y campesinos, Asunción, CPES, 193 p.

GoIRAND Camille, 1998, "Clientélisme et politisation populaire à Rio de Janeiro», Le clientélisme politique dans les sociétés contemporaines, J.-L. Briquet, Sawicki F. éd., Paris, PUF, p. 111-144.

GUASCH Antonio, 1961, Diccionario castellano-guarani / guarani-castellano, Asunción, Centro de estudios paraguayos «Antonio Guasch» (CEPAG).

LÉVI-STrauss Claude, 1955, Tristes Tropiques, Paris, Plon.

— 1965, "Le triangle culinaire », L'Arc, $\mathrm{n}^{\circ} 26$, p. 19-29.

LYNCH John, 1993, Caudillos en Hispanoamérica, 1800-1850, Madrid, Mapfre.

MELIÀ Bartolomeu, 2006, «Escritos guaraníes como fuentes documentales de la historia paraguaya », Nuevo mundo mundos nuevos, $\mathrm{n}^{\circ} 6$.

MontoYa Antonio Ruiz, 1639, Tesoro de la lengua gvarani, Madrid, Juan Sanchez.

RuIz Juan R., 2006, «Figure du cacique, figure du caudillo : les langages de la construction nationale en Espagne et en Argentine, 1808-1930 ", Genèses, n 61, p. 27-48.

Velázquez Rafael Eladio, 1976, "La sociedad paraguaya en la época de la independencia », Revista paraguaya de sociología, Asunción, Centro paraguayo de estudios sociológicos (CPES), vol. 13, n³ 35 , p. 157-169.

19. La thèse citée couvre le champ qui se situe entre ces deux dates mais remonte l'histoire en partant de 2001, date du dernier terrain de l'auteur, jusqu'à 1767 (date de l'expulsion des jésuites). 